\title{
Sepse durante o período gestacional
}

\author{
Sepsis during the gestational period \\ Sepse durante el período gestacional
}

Kamila Lima Abrantes de Matos ${ }^{1 *}$, Luma de Oliveira Morais ${ }^{2}$,Célia Carvalho Cavalcante ${ }^{3}$,Ézio Junio Gonçalves Nunes ${ }^{4}, T_{\text {Talitha Zileno Pereira }}{ }^{3}$, Lenilson Prates da Silva ${ }^{5}$, Francine Ribeiro Potros ${ }^{2}$, Karen Lorrane Maria Antunes Rabelo², Priscila Ribeiro Maia²,Fernanda Miranda Ribeiro².

\section{RESUMO}

Objetivo: Realizar uma revisão de literatura sobre a ocorrência de sepse na gestação, considerando o desafio do diagnóstico e abordagem. Métodos: Foi realizada uma revisão de literatura consultando artigos científicos indexados nas bases eletrônicas LILACS e SCIELO. Resultados: A sepse é a terceira causa de morbimortalidade materna. A gestante tem diagnóstico dificultado devido às adaptações fisiológicas que ocorrem no estado gravídico que confundem-se com os critérios de sepse. As causas podem ser obstétricas ou não, sendo a infecção do trato urinário a principal. O diagnóstico precoce é imprescindível, podendo-se usar sinais clínicos que sugerem hipoperfusão, pois hipotensão e taquipnéia são esperados. $A$ ausculta dos batimentos cardíacos fetais é relevante, pois taquicardia pode ser um sinal precoce de sepse. A prioridade do tratamento deve ser dada à gestante uma vez que o bem estar fetal depende da melhora dos parâmetros maternos. Considerações finais: As mortes maternas por sepse estão principalmente associadas a causas não obstétricas. Atrasos na identificação e no tratamento são importantes no prognóstico materno-fetal e passíveis de intervenção. É necessário alertar sobre as particularidades fisiológicas e critérios que podem ser usados para que haja reconhecimento precoce e melhor condução da sepse na gestação.

Palavras-chave: Sepse, Gravidez, Mortalidade Materna.

\begin{abstract}
Objective: To carry out a review of the literature on the occurrence of sepsis during pregnancy, considering the challenge of diagnosis and approach. Methods: A literature review was conducted consulting scientific articles indexed in the electronic databases LILACS and SCIELO. Results: Sepsis is the third cause of maternal morbidity and mortality. The pregnant woman is diagnosed more difficult due to the physiological adaptations that occur in the pregnancy state, which are confused with the sepsis criteria. The causes may be obstetric or not, with urinary tract infection being the main cause. Early diagnosis is essential, and clinical signs suggesting hypoperfusion can be used, since hypotension and tachypnea are expected. The auscultation of fetal heart rate is relevant, since tachycardia may be an early sign of sepsis. The priority of treatment should be given to the pregnant woman since the fetal well-being depends on the improvement of the maternal parameters. Final considerations: Maternal deaths due to sepsis are mainly associated with non-obstetric causes. Delay in identification and treatment are important in maternal-fetal prognosis and are amenable to intervention. It is necessary to alert about the physiological peculiarities and criteria that can be used for early recognition and better conduction of sepsis during pregnancy.
\end{abstract}

Keywords: Sepsis, Pregnancy, Maternal Mortality.

\footnotetext{
${ }^{1}$ Instituto Brasileiro de Ciências Médicas Juscelino Kubitschek- IBCMED. São Paulo/SP.

*E-mail: kamila.abrantess@hotmail.com

2Universidade Estadual de Montes Claros. Montes Claros/MG.

${ }^{3}$ Faculdade IPEMED de Ciências Médicas. Salvador/BA

${ }^{4}$ Hospital Nossa Senhora da Conceição. Tubarão/SC.

${ }^{5}$ Instituto de Ciências da Saúde - ICS. Montes Claros/MG
} 


\section{RESUMEN}

Objetivo: Realizar una revisión de literatura sobre la ocurrencia de sepsis em la gestación, considerando el desafio del diagnóstico y abordaje. Métodos: Se realizó una revisión de literatura consultando artículos científicos indexados enlas bases electrónicas LILACS y SCIELO. Resultados: La sepsis es latercera causa de morbimortalidad materna. La gestante tiene diagnóstico dificultado debido a lasadaptaciones fisiológicas que ocurrenen el estado gravídico que se confunden com loscriterios de sepsis. Las causas pueden ser obstétricas o no, siendo la infección del tracto urinário la principal. El diagnóstico precoz es imprescindible, pudiéndo se usar signos clínicos que sugierenhipoperfusión, pueshipotensión y taquipnea se esperan. La auscultación de los latidos del corazónfetales es relevante, pues la taquicardia puede ser una señalprecoz de sepsis. La prioridad del tratamientodebe ser dada a la gestante ya que el bien estar fetal depende de la mejora de losparámetros maternos. Consideracionesfinales: Lasmuertes maternas por sepsis se asocian principalmente a causas no obstétricas. El retraso em la identificación y el tratamientoson importantes em el pronóstico materno-fetal y pasibles de intervención. Es necesario alertar sobre las particularidades fisiológicas y criterios que pueden ser usados para que hayareconocimientoprecoz y mejorconducción de la sepsis em la gestación.

Palabras clave: Sepse, Embarazo, Mortalidad Materna.

\section{INTRODUÇÃO}

A infecção no ciclo gravídico-puerperal é uma importante causa na morbimortalidade materna, afetando o binômio mãe-filho. Como definição, é considerada uma disfunção orgânica resultante de uma infecção durante a gestação, durante o parto, pós-parto ou pós-aborto (TORRES R, et al., 2015; WHO, 2017).

A sepse se tornou uma das principais causas de morte materna, responsável por cerca de $15 \%$ dos óbitos. É considerada a terceira maior causa, pois ainda é menos frequente que as causas hipertensivas e hemorrágicas. Nos países desenvolvidos, estas últimas causas diminuíram e possuem protocolos e diretrizes bem estabelecidas, o que ainda não é ocorre frente a quadros sépticos e como resultado representa um maior impacto na morbimortalidade materna (CASTRO EO, et al., 2008; ZASTROW JB, et al., 2017, TORRES R, et al., 2015; WHO, 2017).

A taxa de letalidade de sepse na gravidez e no pós-parto varia de 7 a 12\%. Apesar de sua importância, ainda é considerado um evento raro, provavelmente em decorrência de menor faixa etária destas pacientes, da ausência de comorbidades, sítio da infecção de mais fácil identificação (geralmente a pelve) e que possui facilitado acesso ao foco infeccioso caso haja necessidade de exploração cirúrgica. No entanto, há uma perspectiva de aumento na incidência de sepse na população obstétricabem como na sua gravidade, em decorrência do aumento do número de mulheres que optam por gestar em idades acima de 40 anos, acarretando gravidez de alto risco pela maior chance de comorbidades, principalmente obesidade, diabetes mellitus tipo 2, hipertensão arterial, e de realização de procedimentos invasivos, como inseminação artificial(CORDIOLI RL, et al. 2013; ZASTROW JB, et al., 2017).

As causas de sepse nas gestantes podem ser obstétricas ou não obstétricas. Incluem-se no primeiro grupo causas relacionadas à gestação (aborto infectado, corioamnionite), ao parto (infecções de ferida operatória em cesariana ou em episiotomia, endometrite pós-parto) ou à realização de procedimentos invasivos (infecção pós-cerclagem). No segundo grupo incluem-se infecções de vias urinárias, pneumonia, apendicite, HIV, colecistite, malária. Destas, o principal foco de infecção é o trato urinário, que já é uma região mais predisponente a infecções em decorrência das alterações mecânicas fisiológicas na gestação (BONET M, et al., 2018). A maioria das infecções nas causas obstétricas são de origem polimicrobiana, de foco pélvico ou abdominal, mas, em geral, os principais agentes etiológicos são as bactérias gram-negativas, principalmente E. Coli, e anaeróbios (CASTRO EO, et al., 2008; CORDIOLI RL, et al., 2013; ZASTROW JB, et al., 2017).

Essa revisão de literatura visa abordar os critérios clínicos e laboratoriais, tanto fisiológicos quanto patológicos, que devem ser observados na paciente gestante, com o objetivo de realizar precocemente diagnóstico e tratamento de sepse no período gravídico. É necessário alertar a todos os profissionais médicos que estão menos habituados, principalmente aos que fazem o acolhimento inicial destas pacientes, 
geralmente generalistas, bem como aos intensivistas que as abordarão posteriormente, sobre todas as particularidades do período gestacional para que haja um reconhecimento precoce e melhor condução, pois a qualidade da assistência pode ser um fator decisivo na evolução clínica dessas pacientes.

\section{DISCUSSÃO}

A infecção no ciclo gravídico-puerperal é uma importante causa na morbimortalidade materna, afetando o binômio mãe-filho. Como definição, é considerada uma disfunção orgânica resultante de uma infecção durante a gestação, durante o parto, pós-parto ou pós-aborto (TORRES R, et al., 2015; WHO, 2017).

A sepse se tornou uma das principais causas de morte materna, responsável por cerca de $15 \%$ dos óbitos. É considerada a terceira maior causa, pois ainda é menos freqüente que as causas hipertensivas e hemorrágicas. Nos países desenvolvidos, estas últimas causas diminuíram e possuiem protocolos e diretrizes bem estabelecidas, o que ainda não é ocorre frente a quadros sépticos e como resultado representa um maior impacto na morbimortalidade materna (CASTRO EO, et al., 2008; ZASTROW JB, et al., 2017, TORRES R, 2015; WHO, 2017).

\section{Quadro clínico e diagnóstico}

Os sinais e sintomas da sepse podem ser bem sutis e podem ser mascarados pelas adaptações fisiológicas da gravidez. As manifestações clínicas habituais são hipotensão, taquicardia, taquipneia, hipóxia, alteração do estado de consciência, oligúria (FCCSOB, 2017; BONET M, et al, 2018; RCOG, 2012).

$\mathrm{Na}$ avaliação clínica da paciente obstétrica, o aumento fisiológico da frequência cardíaca, aumento da ventilação e diminuição da pressão arterial podem mascarar os primeiros sinais de infecção grave. A febre nem sempre está presente na sepse, porém um aumento de temperatura deve sempre levar a suspeita de infecção (FCCSOB, 2017).

Um exame físico e anamnese completa podem levar a sinais e sintomas sugestivos de infecção, como dor abdominal ou pélvica, corrimento vaginal fétido e purulento, dor na região do flanco, hiperemia da mama ou sintomas como disúria. A avaliação do estado fetal com a ausculta dos batimentos cardíacos fetais (BCF) é de suma importância, pois taquicardia fetal pode ser um sinal precoce de sepse (CORDIOLI RL, et al., 2013; FCCSOB, 2017).

Os resultados dos exames laboratoriais devem ser interpretados levando em consideração as particularidades, pois a gestante apresenta leucocitose fisiológica, plaquetopenia e alcalose metabólica secundária à taquipneia (FCCSOB, 2017). Essas mudanças podem diminuir a resposta frente a um evento agressor, bem como facilitar a ocorrência de determinados tipos de infecção (CORDIOLI RL, et al., 2013).

Exames de imagem podem ser úteis para identificar a fonte de infecção e a presença do feto não deve impedir o cuidado apropriado com a mãe. Sendo assim, exames com radiação, como radiografia e tomografia computadorizada devem ser usados quando necessário, podendo discutir com o radiologista para minimizar a radiação fetal direta tanto quanto possível, com diminuição da dose de radiação e uso de blindagem (FCCSOB, 2017).

\section{Novas diretrizes de sepse}

As novas definições de sepse e choque séptico foram publicadas recentemente, no ano de 2016, na chamada Campanha Sobrevivendo à Sepse: Diretrizes internacionais para gestão de sepse e choque séptico e o Terceiro Consenso Internacional sobre Sepse (Sepsis-3), nos quais foram formulados e revisados protocolos e diretrizes a fim de reduzir a mortalidade. Com mudanças de conceitos, sepse agora é definida como disfunção orgânica ameaçadora à vida, causada por uma resposta desregulada do hospedeiro à infecção (RHODES A, et al., 2016; BONET M, et al., 2018). Considera-se choque sépticoum quadro séptico com anormalidade circulatória, celular e metabólica associado a um maior risco de morte (RHODES A, et al., 2016). Clinicamente é definido como manutenção da hipotensão, com PAM (Pressão Arterial Média) $\leq 65$ $\mathrm{mmHg}$ e lactato $>2 \mathrm{mmol} / \mathrm{litro}(18 \mathrm{mg} / \mathrm{dl})$ apesar da ressuscitação volêmica adequada, necessitando do uso 
de vasopressores. Configura-se como um choque distributivo. Os termos SIRS (Síndrome da Resposta Inflamatória Sistêmica) e sepse grave não são mais utilizados. Todos os casos de sepse agora são considerados graves (RHODES A, et al., 2016; ZASTROW JB, et al., 2017).

$\mathrm{Na}$ abordagem da sepse é fundamental a sua identificação precoce. Para isso, podem ser utilizados escores de diagnóstico, chamado SOFA (Sequential Organ Failure Assesment - Avalição Sequencial de Disfunção de Órgãos), e de triagem, chamado quick SOFA (qSOFA), uma simplificação do primeiro score. Todavia, as adaptações fisiológicas na paciente gestante não são consideradas na elaboração dos critérios clínicos ou laboratoriais destes scores e, portanto, podem gerar falsos resultados, tanto positivos quanto negativos (RHODES A, et al., 2016).

O score de SOFA é considerado padrão-ouro no diagnóstico de sepse e avalia tanto gravidade quanto prognóstico com base na repercussão clínica nos sistemas (CORDIOLI RL, et al., 2013). No entanto, não apresenta praticidade em seu uso, pois aborda critérios tanto clínicos quanto laboratoriais, que incluem a relação $\mathrm{PaO} 2 \mathrm{FiO} 2$ (pressão arterial de oxigênio/fração inspirada de oxigênio), contagem de plaquetas, bilirrubinas, PAM, escala de coma de Glasgow e nível de creatinina sérica. O score varia de 0 a 4 e quando há dois ou mais critérios presentes detecta-se disfunção orgânica. O SOFA deve ser calculado na admissão do paciente na Unidade de Terapia Intensiva (UTI) e recalculado de 24 em 24 horas (RHODES A, et al., 2016).

$\mathrm{Na}$ gestação, a pressão arterial tende a diminuir no segundo trimestre, podendo levar a uma PAM < $70 \mathrm{mmhg}$ e ser um fator de confusão na avaliação desse score ou ser subvalorizado, pelo fato da hipotensão ser um sinal esperado na gravidez (VELASQUEZ-PENAGOS JA, et al., 2017). É muito tênue o limite entre a normalidade e o critério de sepse. Por esse motivo faz-se necessário o uso de outros marcadores de hipoperfusão clínico e laboratorial, como a dosagem do lactato, que ainda é pouco solicitado ou não disponível em muitos estabelecimentos que acolhem tais pacientes no primeiro momento (VELASQUEZ-PENAGOS JA, et al., 2017).

Da mesma forma, ocorre diminuição de cerca de 10\% da contagem de plaquetas em uma gravidez normal, podendo pontuar no score. A creatinina deve ser $<0,6 \mathrm{mg} / \mathrm{dL}$ devido ao aumento da taxa de filtração glomerular, e no score de SOFA, o valor de creatinina de $1,2 \mathrm{mg} / \mathrm{dL}$ que é considerado normal, já indica comprometimento renal importante na gestante, representando um decréscimo de cerca de $50 \%$ da função renal (CORDIOLI RL, et al., 2013).

O outro score utilizado é o quick SOFA (qSOFA), que apresenta maior praticidade e é utilizado como ferramenta de rastreamento à beira do leito de pacientes que estão fora da UTI com infecção que denota risco elevado de complicações e morte. Este score não define ou diagnostica sepse, porém alerta para alterações importantes que devem ser consideradas para que haja uma abordagem rápida. O qSOFA avalia três sinais clínicos: frequência respiratória >22 incursões respiratórias por minuto, alteração do nível de consciência e pressão arterial sistólica $<100 \mathrm{mmHg}$. Quando há presença de dois ou mais critérios associa-se a um pior desfecho clínico e aumento do tempo de permanência em UTI. Embora seja mais prático que o SOFA por não requerer exames laboratoriais, também não considera as alterações fisiológicas da gestação. Apesar de a grávida não ter seu nível de consciência alterado pelas adaptações da gestação, a taquipneia e a hipotensão atribuíveis ao estado gravídico podem ser um fator de confusão (FCCSOB, 2017; RHODES A, et al., 2016; WHO, 2017).

\section{Tratamento}

O tratamento da sepse baseia-se em diretrizes que foram estabelecidas com base em ensaios clínicos, os quais excluem a população obstétrica. Assim, o tratamento preconizado para os pacientes em geral acaba sendo utilizado nas gestantes (CORDIOLI RL, et al., 2013). Tanto o reconhecimento de sepse quanto o tratamento com ressuscitação e controle da fonte com início da antibioticoterapia devem ser precoces para melhor prognóstico materno-fetal (FCCSOB, 2017).

A gestante deve ser priorizada, avaliando sua resposta clínica pela pressão arterial, frequência cardíaca, saturação de oxigênio, débito urinário e pelas condições fetais, estas melhoradas após a estabilização do quadro materno (CASTRO EO, et al., 2008; FCCSOB, 2017). 
O parto deve ser realizado quando for considerado benéfico para a estabilização materno-fetal, pois sua realização caso a paciente se encontre hemodinamicamente instável pode aumentar a mortalidade (CASTRO EO, et al., 2008). A hidratação materna preconizada no tratamento pode ser o suficiente inicialmente para melhorar o quadro materno-fetal e diminuir a contratilidade uterina. Assim, a resolução da gestação deve ser discutida em ambiente multidisciplinar, considerando estabilidade tanto da mãe como do feto e idade gestacional, sendo a via de parto de indicação obstétrica (TORRES R, et al., 2015)

\section{Fase inicial}

$\mathrm{Na}$ fase inicial do tratamento recomendam-se medidas que devem ser adotadas nas seis primeiras horas, que inclui oferta de oxigênio, hidratação, coleta de culturas para identificar o foco, antibioticoterapia empírica na primeira hora com cobertura polimicrobiana, dosagem de ácido lático e avaliação da diuerese (FCCSOB, 2017; RHODES A, et al., 2016).

O objetivo é conseguir na primeira fase uma PAM $\geq 65 \mathrm{mmHg}$, normalização do estado de consciência, débito urinário superior a $25 \mathrm{ml} /$ hora e dosagem de lactato sérico < 4mmol/litro (CORDIOLI RL, et al., 2013; FCCSOB, 2017; RHODES A, et al., 2016).

Hipotensão é um sinal importante de necessidade de ressuscitação volêmica, porém na paciente gestante, que já apresenta hipotensão fisiológica, o valor de corte deve ser diminuído. Porém, sinais de hipoperfusão como alteração do nível de consciência, oligúria ou elevação do lactato sérico, devem ser considerados uma vez que não são alterados pela gestação. Quando ocorrer suspeita de hipotensão ou hipoperfusão, a paciente deve ter deslocamento uterino para a esquerda com elevação de pelo menos $10 \mathrm{~cm}$ do quadril direito para descompressão da veia cava inferior e aorta, além de indicar necessidade de ressuscitação volêmica (FCCSOB, 2017). O BCF também pode servir como parâmetro para avaliar perfusão (CORDIOLI RL, et al., 2013; FCCSOB, 2017).

$\mathrm{Na}$ ressuscitação volêmica, recomenda-se pelo menos $30 \mathrm{ml} / \mathrm{kg}$ de líquido cristalóide intravenoso nas primeiras 3 horas a fim de reverter a hipoperfusão induzida pela sepse. Após esta medida inicial, a quantidade de fluido adicional deve ser guiada por reavaliações frequentes do estado hemodinâmico (CORDIOLI RL, et al., 2013; RHODES A, et al., 2016).

Porém, como a gestante já apresenta volume sanguíneo aumentado e pressão oncótica colóide diminuída, a reposição agressiva pode levar a edema pulmonar, sendo prudente começar com administração menor de volume e avaliar responsividade aos fluidos (FCCSOB, 2017).

Se ocorre permanência de hipotensão após ressuscitação volêmica, há necessidade de iniciar uso de vasopressor. A droga de escolha é noradrenalina, que tem sido utilizada com segurança na gravidez. A placenta não se auto-regula, portanto a hipotensão materna persistente irá prejudicar a perfusão uteroplacentária e, consequentemente, o feto. A vasopressina não deve ser considerada como primeira escolha, pois causa contrações uterinas. A meta do uso dessas drogas é atingir uma PAM de $65 \mathrm{mmHg}$, porém na população obstétrica não está claro que essa seja a meta apropriada, pois a PAM é normalmente menor (CORDIOLI RL, et al., 2013; FCCSOB, 2017; RHODES A, et al., 2016).

A antibioticoterapia deve ser empírica inicialmente, se ajustando ao resultado das culturas, mantendo duração do tratamento de 7 a 10 dias. O esquema preconizado é ampilicina associado a gentamicina, para infecções como corioamnionite, e clindamicina associado a gentamicina para infecções como endometrite, com o propósito de cobrir bactérias GRAM positivas e GRAM negativas (CASTRO EO, et al., 2008; CORDIOLI RL, et al., 2013; FCCSOB, 2017; WHO, 2015; RCOG, 2012).

\section{Fase de manutenção}

A fase de manutenção compreende controle glicêmico adequado, profilaxia de eventos tromboembólicos, profilaxia de úlcera de estresse e o ajuste do antibiótico ao antibiograma. No caso de choque séptico refratário à hidratação e ao uso de vasopressores, preconiza-se o uso de corticoterapia, com administração de hidrocortisona intravenosa a uma dose de 200mg por dia (CORDIOLI RL, et al., 2013; RHODES A, et al., 2016). 


\section{Complicações}

$\mathrm{Na}$ gravidez, a presença de um feto viável traz importantes considerações maternas e fetais. A infecção pode induzir contrações uterinas, modificações cervicais e conduzir a um trabalho de parto prematuro, além de infecção neonatal (FCCSOB, 2017; RCOG,2012). Outras complicações são: hipóxia, acidose, morte fetal, além de aumentar o número de intervenções por cesariana (CORDIOLI RL, et al., 2013).

\section{CONSIDERAÇÕES FINAIS}

A sepse é a terceira maior causa de morte materna e está principalmente associada a causas não obstétricas. Fatores como atraso na identificação de gestantes sépticas e início tardio da administração de antibióticos são muito importantes no prognóstico e desfecho materno-fetal e são passíveis de intervenção. A falta de protocolos específicos para diagnóstico e tratamento de sepse materna dificultam a abordagem, porém esclarecimentos acerca dos sinais de alerta na gestante e a diferenciação destes com as alterações fisiológicas ciclo gravídico são importantes para que haja suspeita e conduta inicial.

\section{REFERÊNCIAS}

1. AMORIM MMR, et al. Perfil das admissões em uma unidade de terapia intensiva obstétrica de uma maternidade brasileira. Ver Bras Saúde Matern Infant, 2006;6 (Supl 1): S55-S62.

2. BALEDISSERI MR, et al. Fundamental Critical Care Support: Obstetrics. Society of Critical Care Medicine, 2017; Capítulo 5.

3. BONET M, et al. The global maternal sepsisstudy and awareness campaign (GLOSS): study protocol. Reproductive Health. 2018

4. CORDIOLI RL, et al. Sepse e gravidez: sabemos tratar? RevBras Ter Intensiva, 2013;25(4): 334-344.

5. CASTRO EO, et al. Sepse e choque séptico na gestação: manejo clínico. RevBrasGinecolObstet,2008; 30(12):631638.

6. LAKS R, et al. Sepse durante a gestação: Relato de caso. Rev Bras Ter Intensiva, 2007; 19(2): 242-244

7. Rhodes $A$, et al. Campanha Sobrevivendo à Sepse: Diretrizes internacionais para gestão de sepse e choque séptico: 2016. Critical Care Medicine, 2017; 45(3): 486-556.

8. Royal College of Obstetricians and Gyna ecologists (RCOG). Bacterial Sepsis in Pregnancy. 2012

9. SAINTRAIN SV, et al. Fatores associados à morte maternal em unidade de terapia intensiva. RevBras Ter Intensiva, 2016; 28(4):397-404.

10. TORRES R, et al. Maternal sepsis. Centro Hospitalar Lisboa Central - Maternidade Dr. Alfredo da Costa. Acta Obstet Ginecol Port 2015;9(1):65-72

11. VELASQUEZ-PENAGOS JA, et al. Population study of maternal duetosepsis in Antioquia, Colombia, between 2004 and 2014: an overview of the challenge of timely identification and treatment. Ver Colomb Obstet Ginecol, 2017; 68(3):228-238.

12. WORLD HEALTH ORGANIZATION - WHO (Organização Mundial da Saúde). Statement on Maternal Sepsis. 2017

13. WORLD HEALTH ORGANIZATION - WHO (Organização Mundial da Saúde). As recomendações da OMS para a prevenção e o tratamento de infecções maternas no período periparto. 2015

14. ZASTROW JB, et al. Sepse em gestantes atendidas em um hospital público de Curitiba - PR. Ver Soc Bras Clin Med,2017; 16(4):208-211. 